\title{
Discussion on the Bionic Design in Children's Garments
}

\author{
Cao Chunnan
}

\author{
Department of Fine Arts, Qiongtai Normal University, Haikou China
}

\begin{abstract}
Keywords: Children's garment, Bionic design, Market demand
Abstract. The application of bionic design in children's garment can not only develop children's intelligence, but also improve their aesthetic faculties. Mainly focusing on the aspects of color, shape, fabric, the bionic design of children's garments is based on the real condition of children, hoping to improve their intelligence improvement. Regarding the overview of bionic design as an entry point, this article defines the problem existing in the bionic design of children's garment at the present stage, analysis several types of bionic design and puts forward some opinions according the situation, hoping to promote the work of bionic design in children's garments.
\end{abstract}

\section{Introduction}

Bionic design in children's garment, which referred to the design according to some characteristics of animals, has achieved good results. Therefore it is constantly expanding range of applications in babywear, infant's clothing, children's clothing and teenager's clothing and the using frequency and quantity is impressive.

\section{Bionic Design in Children's Garment}

Conceptual analysis on bionic design. Bionic design, also called design bionics, is a new branch of science based on bionics and design, mainly concerning biology physics, psychology, materials science, electronics, etc. It strengthens the application of human imitative capacity mainly based on bionics. In this period, bionic design can be divided into two types: bionic art design and bionic engineering design. During the research stage, it mainly focused on natural beauty. Then people's felling integrated with the adaption between subjective factor and objective, which makes the goods attract people's attention and promotes the consumption. Bionic art design can be divided into industrial bionic design, environmental bionic design, garment bionic design etc. Object is the core of engineering bionic design, which means constant innovation within some complete objectives.

Present Analysis on Bionic Design in Children's Garment Home and Abroad. (1) Current situation of domestic bionic design in children's garment. There are a lot of research and information reports about children garment industry in China. Some indicate the scale and gathering places of this industry in China in details, offering reliable industrial information to garment researchers. Supported by this information, research segmentation, such as the garment bionics can grasp the whole picture easily. Now the domestic bionic design in children's garment mainly appears on the stage or some children photography agencies and is rarely seen in public, which is the results of its exaggeration. On the basis of some animals, trees and flowers, this kind of design is mainly to render stage effect. Although the designed appearances are vivid, lovely and gentle color matching, they are complicated to wear with bad practicability. Meanwhile, the existing brands of bionic design are less, which makes the children lose naivety when wearing this kind of clothes. Therefore, the bionic design in children's garment in China is still lagging.

Current situation of bionic design in children's garment abroad. The overseas children's garment brands are bullish on Chinese market and have already held a certain market share. They are relatively more mature than China, but the children's garment brands specialty in bionic design are still rare. Most of them are common bionic patterns and are very adult. It is undeniable that the overseas brands cover more expanding space and they grasp the consuming psychology of Chinese young parents, occupying more garment market in China gradually. With the development of economy, the high-end consumers are expanding. The increase of consumption ability and love of 
them for high-end apparel products provide good opportunity overseas brands entering into Chinese market, which makes them come into view of Chinese consumers and cover quite large market share. The consumer need for children's garment trends to fashion and branding.

Theoretical Analysis on Bionic Design in Children's garment. The combination of children's garment and bionic design is of artistry, enjoyment and rigidity principle. Different with other children's garment design, it only collects various high-tech functional fabrics, but also pays more attention to its special design principle, such as safety, comfort, functionality, creativity and enjoyment. Meanwhile, children's garment and bionic design principle have great effect on child's body and mind.

Characteristic and principle of bionic design in children's garment. First, based on bionics, bionic design in children's garment is according to the animals and plants in nature. The design usually directly restores creature prototype or on the basis of the characteristics of unique biological changes and detailed imitation, alters part of the garments. Secondly, the design inspiration is always based on creatures with spectral characteristics. For example, a more lively appearance can make a person who under great pressure feel happy. This kind of clothes can bring special enjoyment and create an environment of consuming. More funny and happiness factors are added into the design. The design of children's garment need more lively factors because the pursuit and even the need of relax is increasing. Last, bionic design is of strong entertainment, which referred to the feeling produced in people's heart and brings people different experience. What's more, the design is not copying the whole appearance of the creature, but makes creative design by exaggerated modelling, color matching, material usage, etc., promoting the pursuit of entertainment in a certain degree.

Bionic design in children's garment. Security principle. In the design of children's garment, safety attaches equal importance to exaggerated appearance. Comfort principle, which referred to the positive attitude in children's garment functional design, is the way to cater to the market and occupy more market share. Functional principle. It is an important principle that designers should choose suitable ultraviolet-proof antistatic fabric because of children's weak resistance, guaranteeing every aspects satisfied the need of bionic design. Creative principle. It is the key to pretend rigid design.

\section{Application of Bionic Design in Children's Garment}

Application of bionic pattern in children's garment design. The application of bionic pattern in children's garment can be divided into three parts: (1) the bionic application of concrete patterns. Directly using the intuition and vitality of some natural creatures, this kind of bionic design transforms then into concrete patterns, bringing people aesthetic feelings. If it can be used into the design of children's garment, the garments will be more vivid and are close to children's real life. The inspiration of pattern design is from nature. It is not a complete copy of nature, but an integration of art through author's conception. By researching and using some concrete objects, the designers break the limit of image set and find it characteristics. Relying on different images, redesigning can gain great effects. (2) The analysis of abstract pattern bionic design. Abstract patterns mainly refer to some simple specific objects separated with practice which is hard to comprehend, but it usually comes out of some specific things in daily life. In general, abstract patterns can reflect physically spirit, showing the emotion of the author. In addition, they are also a kind of unconscious perceptual expression way, belonging to relative existence. There exists certain differences between abstract patterns and origin patterns, resulting in a flexible design style. Because there are no exact bound among those patterns, mix-and-match can be used in the design of children's garment, ensuring the conciseness and layering of pattern design. (3) The analysis of three-dimensional pattern. Three-dimensional structure is the main component factor of three-dimension pattern design and is also the fundamental guarantee of good wearing effect. The application of three-dimension patterns can make the design more vivid thus satisfying the market need.

Applied analysis on fabric bionic in children's garment. The bionic design in children's garment is not only a combination of practicability and artistry, but also design demand with rigidity principle. It should compare with the bionic standard timely in the aspect of details, patterns, color 
and modelling, ensuring the contents can satisfy the real job requirements. What's more, not only some high-tech functional and comfortable fabrics should be used, some special fabrics should also be controlled, ensuring good adaptability and plasticity.

Adaptability analysis on bionic fabrics in children's garment. The positive attitude to the research of bionic fabric adaptability has great significance and it is also the fundamental and soul of the design work. First, the chosen fabrics should have good safety and comfort and are also up to the standard of Chinese children's garment design. For example, in the chemical aspect of fabric, the quantity requirement of 6 kinds of plasticizer, lead and cadmium is added, which can improve the comfortableness of children's garment fabric. Secondly, in order to prevent untoward effect in the process of wearing clothes, hygroscopicity and breathability should be considered when choosing children's garment fabrics. Thirdly, good anti-static function and good plasticity of the fabrics should be guaranteed. The clothes which can generate static electricity are easily to absorb the dust in the air and are of weak plasticity, which will cause certain influence on human body. With good anti-static function and plasticity, the bionic fabrics can make the garment keep high degree of comfort. What's more, owing to good plasticity, the designed garments have great vision effect. Therefore, the comfort of bionic fabrics affect the whole design of children's garment directly.

Plasticity research of bionic children's garment fabrics. Style, color, fabrics are the main component factors during the clothes designing process and the quality and plasticity of fabrics has direct impact on color and style on certain conditions. Therefore, the plasticity plays an important role in bionic design of children's garment. If the quality of the fabrics is good, the clothes will be dignified, vivid and affinity. First, the crisp fabric can be used to produce diversity appearances and most of them are exaggerated. Second, with heavy texture style, fluffy fabric brings a sense of expanding. The inside and outside can be integrated with each other to make perfect children's wear. As for some shorter fluffy fabric, the designer can adjust the structure according to real situation, which can improve the enjoyment of design and cater to the market demand.

Restrictive research of bionic children's garment fabrics. Not all fabrics can be used in bionic design in the real design process because of various reasons. Some are too soft to support the design appearance and can't show the design philosophy exactly, such as real silk, silk spinning etc. They are not easy to be shaped of its smooth and soft texture, so they are seldom used in application process. Some transparent fabrics are also not adopted because of its poor safety performance and weak plasticity. Different fabrics adopt different raw materials and processing methods, the various performance not only has an effect on appearances, but also on the structure of the clothes. Clothes version is the fundamental guarantee of a successful design, so designers should pay much more attention to it.

Application of color bionic in children's garment. Color matching is an important component factor in garment design. Generally speaking, garment color are with strong era sensation and the represent color of different ages are not the same.

(1) Bionic a single color is an easy way in the application process. The so-called single color mainly refers to red, orange, yellow, green, blue, indigo and purple. Different colors bring different visual experience. In the real bionic application process, when designer confirms bionic objects, they should research and analysis the origin colors of bionic creatures.

Using the creatures' origin colors directly is not as easy as people think. Analysis should be made first. Designers should simplify the relevant colors and find the most characteristic one, expanding it to recognize the prototype characteristic most. The cognition of a certain creature is accumulated in daily life by virtue of people's own experience and memory. Only copying the original colors will not only produce a bad feeling, but also make the design skin-deep. Therefore, complicated creature colors should be extracted and then matched, ensuring it will show the final effect of physical beauty. This can not only ensure the primary aberration, but also strengthen aesthetics.

(2) Research and analysis of polychromatic collocation bionics

Polychromatic collocation bionics refers to extract the colors of animal prototypes, choosing some characteristics and colors with strong representation to utilize comprehensively. Polychromatic collocation bionics is not a mechanical copy, but a way to draw upon some colors of the creature. 
Children themselves should also be considered, ensuring the clothes can show the lovely side of children. The color application is wider and different color matching can imitate different creature prototypes. Emotion should be brought into the actual matching process to achieve better results. This is just what single color does not have. Choosing the number of color matching and the way of matching all have direct impact on overall effect because the characteristic of creatures are different. Generally speaking, one or two major colors and several secondary colors will be set. According to this way, the designed work will not only have better visual effect, but will also show more vivid appearances.

Application analysis of technical bionic design in children's garment. The children's garment design is a whole design flow with certain systematization. The prototype selection, color matching selection and the final accomplishment are all the embodiment of design and technology. Any style design should be confirmed by following the relevant clothes designing process directly, which will strengthen the control of each step, improving the quality and standard of design fundamentally.

Analysis of technical details. The quantity and types of clothes are larger in children wear market. With the improvement of people's living standard, people have more requirements of children wear. They not only want a more novel style, but also have high standard for fabrics and workmanship. Therefore, during the bionic design process, focusing on the technical details actively is of great significance. Technical details of each clothes is made gradually by designers' comparison and improvement.

Analysis of technical difficulties. Some basic technology is the fundamental guarantee of children's wear quality and the basis of bionic design in children's garment. Generally speaking, it requires high level of overall operation for designers because the structures of some steps are complex and more details and problems need to be focused during actual working process. Therefore, there exists a lot of difficulties in adopted technology. In the aspect of structure, certain particularity exists between bionic children's wear and its prototype and the cutting line of them is hard to be confirmed, which is difficult to make children's garment more vivid and achieve better bionic effects. What's more, the matching of different materials is difficult. The joint and integration of design styles, colors and materials together make up the bionic design in children's garment. But if the matching of different elements is unreasonable, the form of bionic biological prototypes can't be achieved. Last, it is necessary to perfect and adjust relevant work actively, thus ensuring products can satisfy consumers' actual demands. In addition, designers should continuously improving themselves, take the successful experience, control every product actively and strengthen the details of every aspects, so a better design effect can be achieved.

Analysis of technical structure. Technical structure is the core part the garment design. Only control it actively, can design quality be improved. Generally speaking, garment structure has direct impact on the whole version and it graphic design of garment making. On account of relevant making materials and technology are different, we should not only consider the aesthetics, but also consider the integration of each part when we mix the relevant technologies. Only when it passes audits, large scale production can be made.

\section{Conclusion}

In conclusion, bionic design offers a brand-new development direction for children's garment design during the development process of children wear industry, which makes it significant in researching and analyzing bionic design in children's garment. Only confirm the application pattern bionics, the application material bionics, the application of color bionics and the application of technical bionics and improve the management of each step, can the work be promoted.

\section{References}

[1]Wen Li, Zhang Zhichun. Brief discussion of bionic design in children's garment [J]. Journal of Nanning Vocational \& Technical College, 2006,02:20-22. 
[2]Zhang Jing, Cai Hong, Luo Qianqian. Creative exploration of bionic design in puzzle children wear [J]. Liaoning Tussah Silk, 2014, 01:8-9.

[3]Liu Huan. Research of children wear bionics based on early children mental [J]. Art Panorama, 2012,12:145.

[4]Zhou Xiaoru, Yao Mengyuan. The application of bionic design in children's apparels [J]. Art Panorama, 2012,12:145.

[5]Liang Yalin, Chen Yu. Style research of green design in children's garment [J]. Art \& Life, 2011,03:66-68. 